\title{
Frequency and Phase Modulation Performance of an Injection-Locked CW Magnetron
}

\author{
Imran Tahir, Amos Dexter, and Richard Carter, Senior Member, IEEE
}

\begin{abstract}
It is demonstrated that the output of a $2.45-\mathbf{G H z}$ magnetron operated as a current-controlled oscillator through its pushing characteristic can lock to injection signals in times of the order of 100-500 $\mathrm{ns}$ depending on injection power, magnetron heater power, load impedance, and frequency offset of the injection frequency from the natural frequency of the magnetron. Accordingly, the magnetron can follow frequency and phase modulations of the injection signal, behaving as a narrow-band amplifier. The transmission of phase-shift-keyed data at $2 \mathrm{Mb} / \mathrm{s}$ has been achieved. Measurements of the frequency response and anode current after a switch of phase as a function of average anode current and heater power give new insight into the locking mechanisms and the noise characteristics of magnetrons.
\end{abstract}

Index Terms-Amplification, frequency shift keying (FSK), injection locking, magnetrons, modulation, phase-locked loops (PLL), phase shift keying (PSK), twining.

\section{INTRODUCTION}

$\mathbf{T}$ HIS PAPER gives time-domain measurements and analysis of the response of an injection-locked continuouswave $(\mathrm{CW})$ "cooker" magnetron to changes in the phase and frequency of its injection signal. Effectively, the magnetron is being operated as a narrow-band reflection amplifier [1]-[3]. Recent work by the authors has shown how injection power can be minimized and operation stabilized in the presence of considerable power supply ripple and load fluctuation by precise control of the anode current [4]. The output frequency the magnetron would have without injection is kept close to the injection frequency by utilization of the magnetron's pushing characteristic. Our technique differs from that described by Shinohara et al. [5] in that current control is tightly integrated into the control circuits of the switch-mode power supply. The reflection amplifier described here achieves $27-34 \mathrm{~dB}$ of amplification for phase-shift-keyed (PSK) information at data rates up to $2 \mathrm{Mb} / \mathrm{s}$ with no bit errors for large data files $(\gg 1 \mathrm{Mb})$. The ability of an injection-locked magnetron to follow rapid phase reversal with fidelity and without noise degradation has previously been reported by Weglein and Leach [6]. Studying the response of the magnetron to a changing injection signal as a function of heater power, injection level, anode current, and load impedance gives new insight on the locking mechanism, magnetron noise performance, and twining.

Manuscript received January 5, 2006; revised April 19, 2006. This work was supported in part by the Particle Physics and Astronomy Research Council, U.K., and in part by e2v Technologies Ltd. The review of this paper was arranged by Editor W. Menninger.

The authors are with the Department of Electrical Engineering, Lancaster University, Lancaster LA1 4YR, U.K. (e-mail: a.dexter@lancaster.ac.uk).

Digital Object Identifier 10.1109/TED.2006.876268
Significant departures from the phase response predicted by the Adler equation are identified for regimes of space-chargelimited emission. The work also gives data in accordance with Brown's explanation of certain aspects of magnetron noise [7], [8]. Brown describes two noise states of the cooker magnetron, namely 1) a low-noise state when emission from the cathode is temperature limited and 2) a high noise state when emission from the cathode is space charge limited, i.e., the filament temperature is higher than it needs to be. He determined excess emission and hence the point of temperaturelimited emission by applying a step voltage to the anode [9] and observing the magnitude of the initial step change in the anode current. Measurements here show how the phase settles after a $90^{\circ}$ shift as a function of heater power. The measurements here and the measurements of Brown relate primarily to closeto-carrier noise. As early as 1977 , close-to-carrier phase noise for 25 to $50 \mathrm{~W}$ positive-anode military magnetrons had been reported to be very low, with frequency-domain measurements published by Garrigus [10] implying a root-mean-square (rms) phase jitter of about $4^{\circ}$.

This paper utilizes cooker magnetrons (so named because they are found in commercial microwave cooking ovens) rather than military magnetrons. An excellent review of noise spectra from cooker magnetrons is given by Osepchuk [11]. Recent noise data for a cooker magnetron driven from a half-wave rectified source has been given by Mitani et al. [12]. New techniques to reduce noise from magnetrons at start up and when driven from half-wave sources are of current interest [13]-[15].

The ultimate aim of this paper is to investigate whether magnetrons can be used to drive high- $Q$ cavities for long pulse and $\mathrm{CW}$ accelerator applications. An important aspect of driving accelerator cavities is the need to compensate for detuning factors such as vibration. Any practical RF system for an accelerator must be able to vary its phase on a relatively short timescale as required to maintain the phase of the accelerator cavity with respect to charged bunches in the accelerated beam. The motivation for investigating magnetrons for accelerator applications is that the capital outlay per kilowatt for producing $\mathrm{RF}$ in the gigahertz frequency range using magnetron technology is much less than that for producing RF with klystrons or inductive output tubes (IOTs). Minimization of cost is likely to be crucial for future high-energy-physics accelerators. The cost savings that magnetrons offer come from their smaller size, their higher efficiency, and their lower anode voltage. The smaller size gives big cost savings for the magnet. The lower anode voltage greatly reduces the cost of the power supply. Where multiple sources are needed to drive an accelerator, phase control is crucial. Reasons cited against the use of magnetrons 


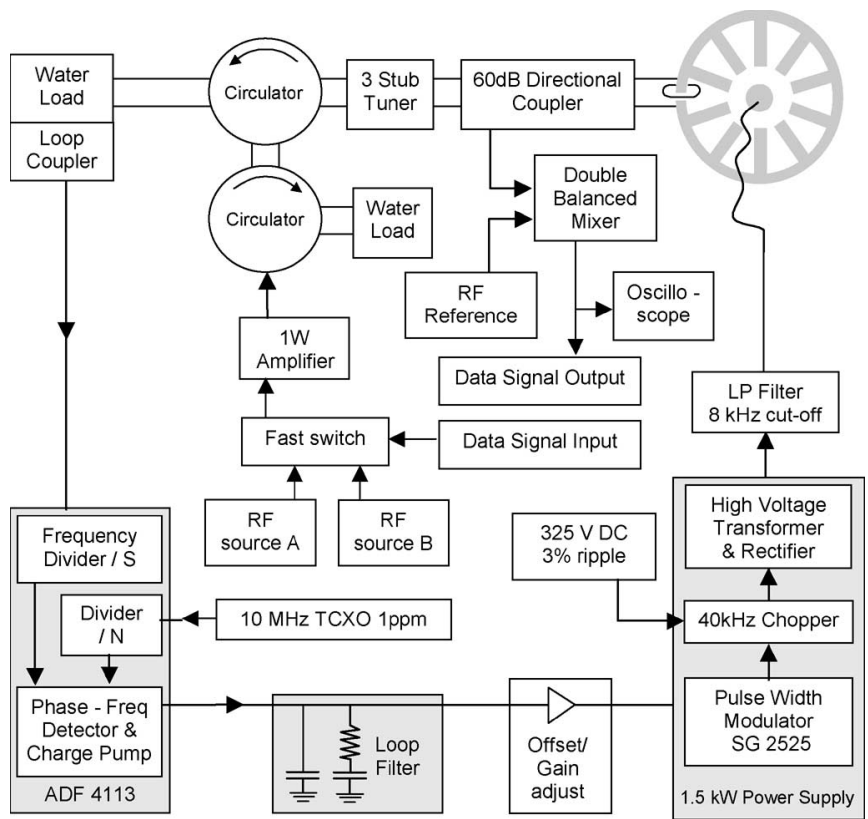

Fig. 1. Experimental arrangement.

are the large amounts of injection power required for locking poor noise performance and narrow instantaneous bandwidth. Recent work investigating the applicability of phase-locked magnetrons to driving accelerator cavities focused on shortpulse and high-power technology [16]-[18]. This paper is also applicable to scalable power supplies for industrial processing.

\section{EXPERIMENTAL ARRANGEMENT}

The experimental arrangement is shown in Fig. 1. The magnetron used for this work was a 1.2-kW CW Panasonic 2 M137. The current to the magnetron is supplied by a switch-mode power supply. The chopper pulsewidth controls the output current of the power supply. Rather than operating the power supply at constant current, the current is varied to keep the magnetron running at close to a steady frequency (even in the absence of the injection signal). This is possible as the frequency the magnetron would have in the absence of a locking signal is a function of the magnetron current. Current to the magnetron is thereby controlled by a phase-locked loop (PLL). Phase frequency detection for the PLL was implemented with a low-cost ADF 4113 IC. The output of this chip is a series of current pulses whose length is proportional to the phase difference. The measured phase difference can be up to $2 \pi S$, where $S$ is the division ratio of the input signal. The absolute reference was provided by a 1-ppm $10-\mathrm{MHz}$ temperature-compensated crystal oscillator. The division ratio $N$ for the reference signal was set as $\mathbf{5 0}$ for most of the work, thereby permitting PLL control frequency adjustments in steps of $200 \mathrm{kHz}$. The division ratio $S$ is then determined as the required magnetron output frequency divided by $200 \mathrm{kHz}$.

The loop filter acts as a proportional/integral controller and hence determines how the switch-mode power supply's output varies in response to a phase error. The loop filter was optimized for magnetron anode currents in the range of $180-380 \mathrm{~mA}$. The loop filter needs a differing optimization dependent on

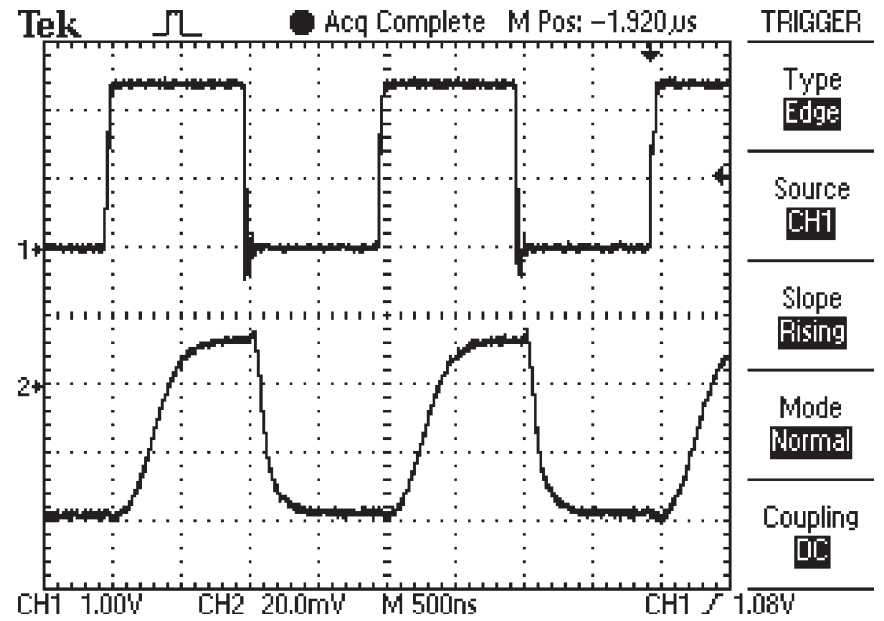

Fig. 2. PSK modulation signal (upper trace) being applied to a $1-\mathrm{W}$ microwave injection signal and the associated demodulated magnetron output, i.e., the magnetron phase (lower trace) for a cathode heater power of $10 \mathrm{~W}$.

the gradient of the pushing curve [4]. Frequency control of the magnetron by the technique described ceases to work where the gradient of the pushing curve is zero.

The injection signal is shown in Fig. 1 as being derived from one of two RF sources via a fast pin diode switch and fed to a 1-W amplifier. For the demonstration of PSK, the RF source B was derived from source A with an adjustable phase shifter. Independent sources were used for the demonstration of frequency shift keying (FSK). These sources were derived from voltage-controlled oscillators (VCOs) controlled by separate ADF 4113 ICs. A double-balanced mixer was used to demodulate signals amplified by the magnetron.

It should be noted that the ripple performance of the $325-\mathrm{V}$ dc supply has been improved from 5\% to $3 \%$ by addition of extra smoothing capacitance with respect to previously reported work [4]. This new ripple performance is still consistent with a low-cost switch-mode power supply.

\section{PSK MODULATION}

Fig. 2 shows on its upper trace a data signal going to the fast switch in Fig. 1. The lower trace of Fig. 2 shows a snapshot of the output from the double-balanced mixer on an oscilloscope. The figure relates to an injection frequency of $2.4519866 \mathrm{GHz}$, an injection power of $1 \mathrm{~W}$, switching between two phases $90^{\circ}$ apart at $0.5 \mathrm{MHz}(=1 \mathrm{Mb} / \mathrm{s})$ and a heater power of $10 \mathrm{~W}$.

The control frequency for the PLL that adjusts current through the magnetron anode was also set to $2.4519866 \mathrm{GHz}$. The microwave signal input to the double-balanced mixer was sampled from the magnetron output waveguide with a $60-\mathrm{dB}$ directional coupler. Fig. 2 shows the magnetron adjusting its output phase to that of the 1-W injection signal in about $500 \mathrm{~ns}$. Switching times were found to depend on the injection power, the heater power, the anode current, and the impedance of the load, i.e., the magnitude and phase of power reflected back to the magnetron.

When the magnetron is turned off while the injection signal is still present, the step voltage between the phases measured by the double-balanced mixer falls by about $30 \mathrm{~dB}$. This fall relates to signal gain achieved by the magnetron. More precise 


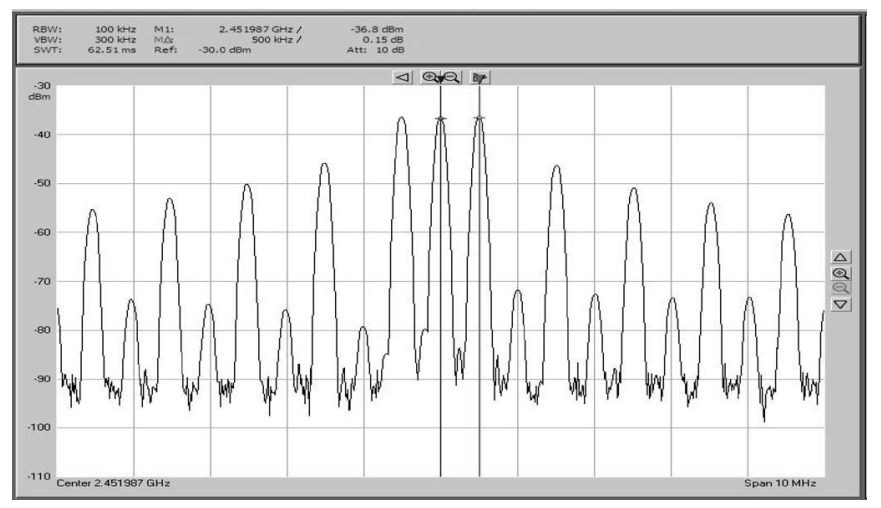

Fig. 3. Spectrum of the magnetron injection signal associated with data signal in the upper oscilloscope trace of Fig. 2 that switches the injection signal's phase.

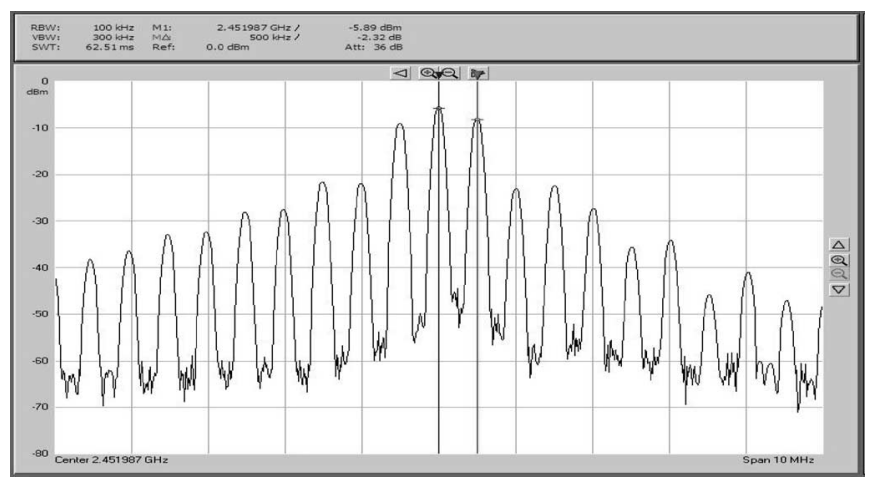

Fig. 4. Spectrum for magnetron output corresponding to the lower oscilloscope trace in Fig. 2.

measurements of gain have been made with a spectrum analyzer. The bandwidth of the magnetron output before injection locking is about $500 \mathrm{kHz}$ and depends in part on the dc power supply ripple. The bandwidth for the magnetron locked with a steady injection signal is very small, and the output frequency perturbations associated with $50-$ and $100-\mathrm{Hz}$ ripple form distinct sidebands in the spectrum [4].

When the injection signal is given a regular PSK modulation, its spectrum acquires new bands shifted from the center injection frequency by the frequency of modulation. Fig. 3 shows the spectrum of the input injection signal to the magnetron as associated with the modulation for the upper trace in Fig. 2. Fig. 4 shows the spectrum for the magnetron output corresponding to the lower trace in Fig. 2. Note that the input reference level for Fig. 3 is $-30 \mathrm{dBm}$, whereas the input reference level for Fig. 4 is $0 \mathrm{dBm}$.

For a perfectly square modulation with equal periods of $90^{\circ}$ phase-shifted output, the three central peaks in the spectrum will have the same height as can be seen in Fig. 3. As the time required for the new phase to establish itself increases, then the heights of the two sidebands on either side of the center band are reduced. Shutting down the magnetron with the injection signal carrying data still applied and measuring the reduction in the height of the sidebands determines the effective amplification of signal information by the magnetron. For a $90^{\circ}$ phase shift at $1 \mathrm{Mb} / \mathrm{s}$ on a $1-\mathrm{W}$ locking signal and for a magnetron heater power of $10 \mathrm{~W}$ and anode current of $200 \mathrm{~mA}$, a $27-\mathrm{dB}$ reduction in the sidebands was observed when the magnetron was deenergized.

\section{Dependency on Heater Power}

Fig. 2 showing the phase switching time for one specific operating point was taken as a snap shot. As the magnetron switching time has a dependence on the heater power, it therefore has a dependence on the $50-\mathrm{Hz}$ heater power ripple. The switching time also depends on the magnetron anode current and hence depends on the $100-\mathrm{Hz}$ power supply ripple. The double-balanced mixer output showing phase as a function of time has therefore both $50-$ and $100-\mathrm{Hz}$ jitter.

The dependence of switching time on heater power and anode current are displayed most clearly when the doublebalanced mixer output is averaged over multiples of $20 \mathrm{~ms}$. Using averaged outputs, Fig. 5 shows the demodulated magnetron output into a matched load as a function of heater power. Because the center frequency of the magnetron output is fixed, the anode current variation with heater power is seen.

The response to the change in phase has the appearance of a second-order system going from being over damped for low heater powers to being under damped at high heater powers. This transition depends on the anode current with respect to the heater power. Working at anode currents near to $220 \mathrm{~mA}$, the transition is seen most clearly. At higher anode currents, corresponding to higher frequencies, the oscillation at high heater powers is less pronounced (figures not shown). Fig. 5 is also asymmetric with respect to the direction of the phase change. In the figure, the upper voltage level corresponds to the advanced phase, whereas the lower voltage level corresponds to the retarded phase. It can be seen that retarding the phase (switching down) is more responsive than advancing the phase.

The switching response has also been investigated for unmatched loads. For small amounts of reflected power at phases between $45^{\circ}$ and $105^{\circ}$ toward the load from the point on the Rieke diagram of maximum efficiency, the oscillations at higher heater currents and relatively low anode currents (about $220 \mathrm{~mA}$ in this case) are almost undamped. Fig. 6 shows magnetron switching response as a function of heater power for a load impedance that reflects $13 \%$ of the power back to the magnetron at a phase of approximately $100^{\circ}$ toward the load from the phase on the Rieke diagram yielding maximum efficiency. The last trace in Fig. 6 shows the weakly damped oscillations.

The response was also measured for $\sim 10 \%$ reflected power at phase intervals of $15^{\circ}$ over the full $360^{\circ}$. Figs. 5 and 6 broadly encompass the range of characteristic response.

Light can be cast on possible mechanisms that explain the switching behavior by considering the anode current during switching and also pushing curves as a function of heater power. Figs. 7 and 8 show the magnetron anode current referenced to the magnetron phase as it switches. The figures have been determined for differing injection frequencies and hence have differing currents. (The PLL control frequency was the same as the injection frequency in each case.)

The figures show that in regions where the phase gets retarded, the magnitude of the anode current reduces, then 


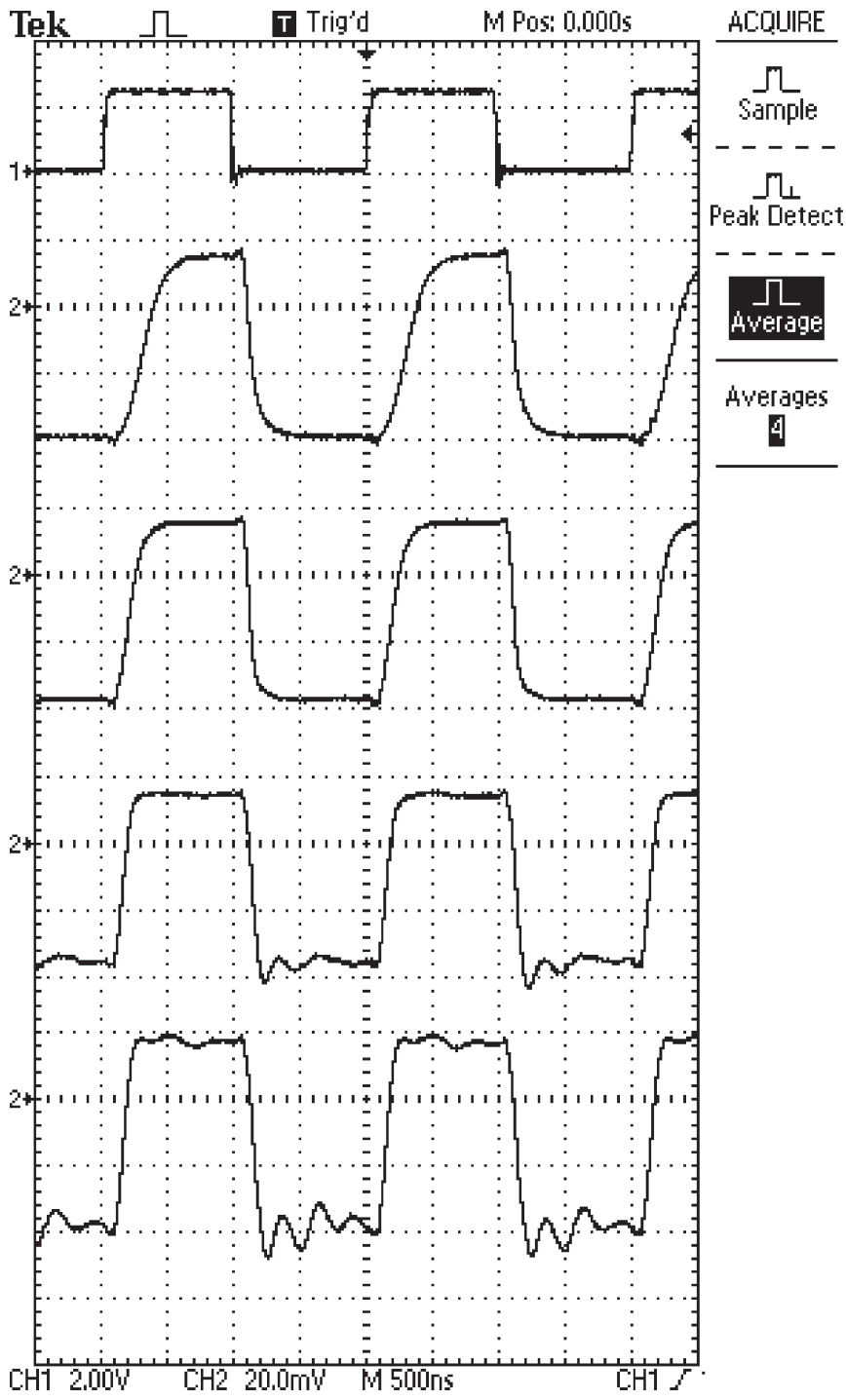

Fig. 5. PSK modulation signal (upper trace) being applied to a $1-\mathrm{W}$ microwave injection signal and the averaged demodulated magnetron output phase (lower four traces) for cathode heater powers of 8, 15, 36, and $43 \mathrm{~W}$ in downward order. Corresponding anode currents were 207, 210, 225, and $265 \mathrm{~mA}$. The magnetron operates into a matched load, frequency $=2.4504 \mathrm{GHz}$.

increases again, overshooting the average value before settling back. For both Figs. 7 and 8, the reduction is about $100 \mathrm{~mA}$. Note that downward segments of the magnetron phase traces correspond to the phase retarding, and the humps in the anode current traces correspond to reductions in anode current magnitude. The figures also show that in regions where the phase advances, the anode current increases but settles back without overshooting. In Fig. 7, the increase in current is about $40 \mathrm{~mA}$, whereas in Fig. 8, it is about $60 \mathrm{~mA}$. It will be seen later that the current variations correspond in part to the magnetron output frequency perturbations needed to advance or retard the phase.

\section{Discussion of THE LOCKING MECHANISM}

For the magnetron to respond to a change in phase of the injection signal, it must either increase or decrease its output frequency until the phases are synchronized again. The estab-

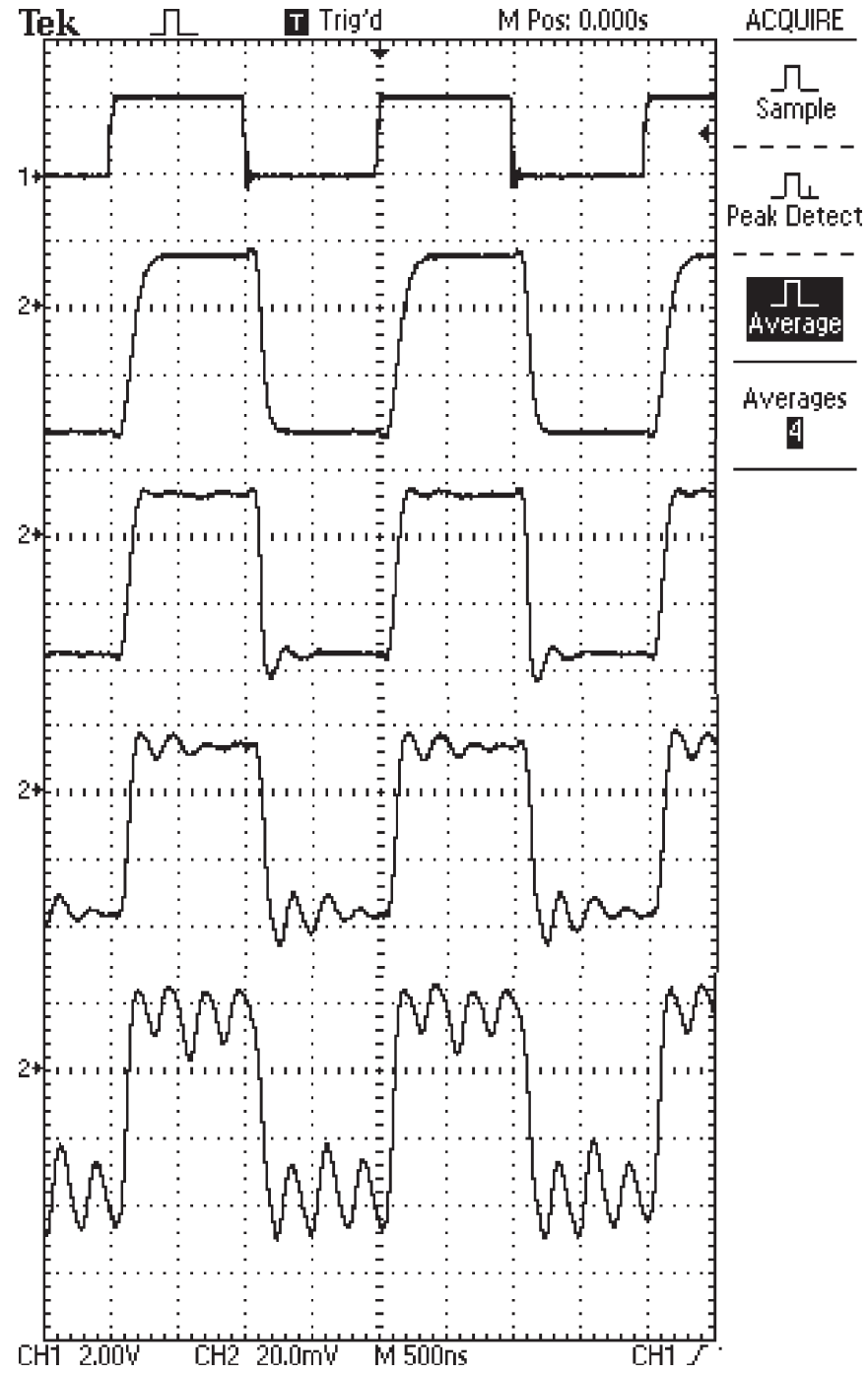

Fig. 6. PSK modulation signal (upper trace) applied to a 1-W injection signal and the averaged demodulated magnetron output phase (lower four traces) for cathode heater powers of $8,15,36$, and $43 \mathrm{~W}$ in downward order. Corresponding anode currents were 222, 201, 217, and $265 \mathrm{~mA}$. Mismatch $\sim 13 \%$ reflected power at $100^{\circ}$ toward the load, frequency $=2.4520 \mathrm{GHz}$.

lished theory of magnetron injection locking [19], [20] considers only the pulling effect of the injection signal, i.e., the injection signal changes the effective load impedance. This locking mechanism can be explained with a simple parallel lumped circuit model for the magnetron as utilized by Slater [21]. Following Woo et al. [20], the lumped circuit analysis yields the differential equation

$$
\ddot{V}-\frac{\omega_{o}}{Q_{L}}\left(\frac{Y_{e}}{Y_{L}}-1\right) \dot{V}+\omega_{o}^{2} V=\frac{\omega_{o} \omega_{i}}{Q_{L}} V_{\mathrm{inj}} \sin \left(\omega_{i} t\right)
$$

where $V$ is the equivalent circuit voltage, $V_{\text {inj }} \cos \left(\omega_{i} t\right)$ is the injection voltage, $\omega_{i}$ is the injection frequency, $Y_{e}$ is the electronic admittance of the space charge, $Y_{L}$ is the admittance of the anode circuit together with the load, $Q_{L}=\left(1 / Y_{L}\right) \sqrt{C / L}$ is the loaded $Q, L$, and $C$ give the equivalent inductance and capacitance of the anode circuit, respectively, and $\omega_{o}=$ $(1 / \sqrt{L C})$. The electronic admittance decreases as the power output increases, until at full power, the negative resistance term 


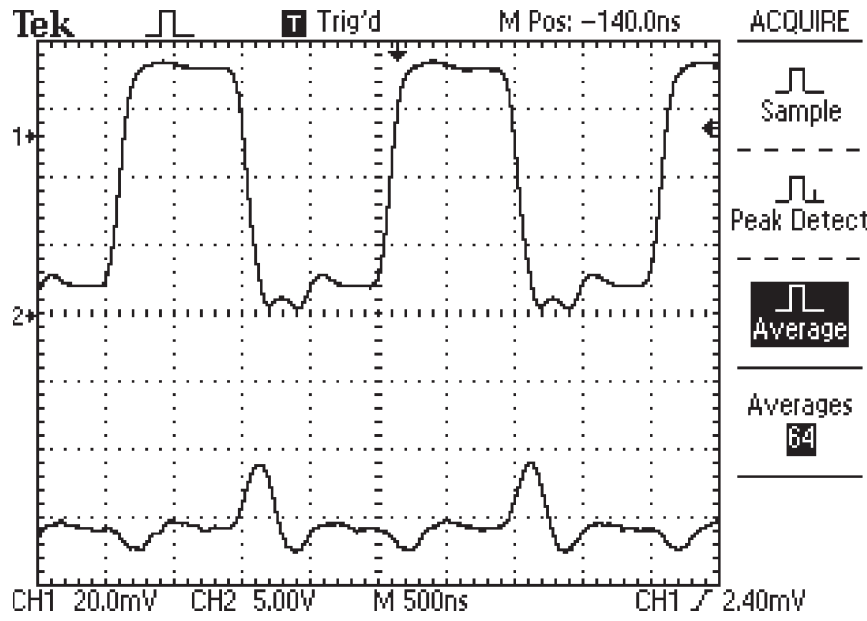

Fig. 7. Anode current (lower trace) referenced to the magnetron phase (upper trace) for $90^{\circ}$ phase shifts, a heater power of $43 \mathrm{~W}$, an injection power of $1 \mathrm{~W}$, and a matched load. The scale for the lower trace (the anode current) is $20 \mathrm{~mA}$ per tick mark, and its zero is marked on the left-hand scale with a 2. The average anode current (measured independently from scope trace) $=$ $-330 \mathrm{~mA}$, frequency $=2.4512 \mathrm{GHz}$.

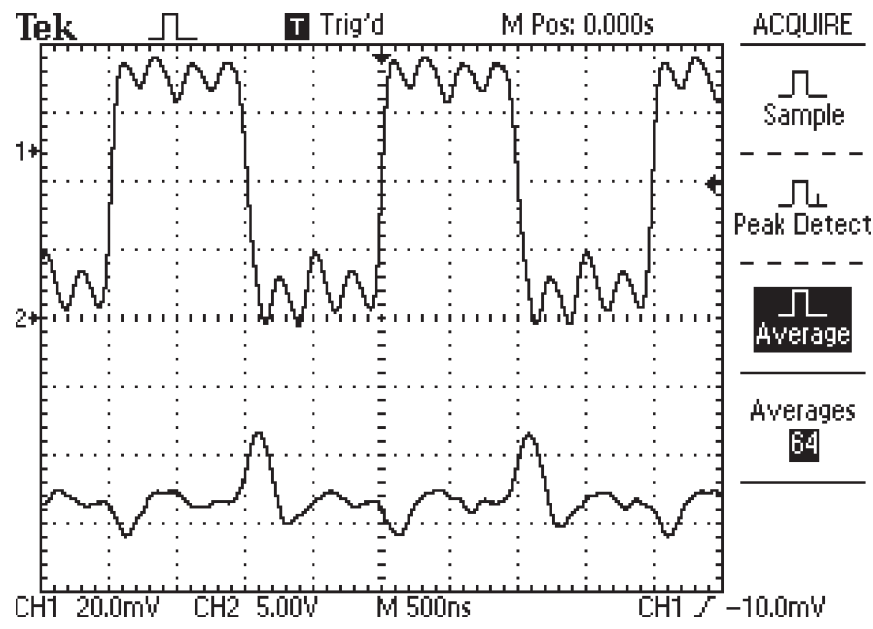

Fig. 8. As Fig. 7 except for the load, average anode current $=-285 \mathrm{~mA}$, frequency $=2.4522 \mathrm{GHz}$.

$\left(\omega_{o} / Q_{L}\right)\left(\left(Y_{e} / Y_{L}\right)-1\right)$ vanishes. One then sees that $\omega_{o}$ is the steady-state frequency in the absence of an injection signal. When an injection signal is applied, the steady-state solution becomes

$$
V(t)=V_{\mathrm{RF}} \cos \left(\omega_{i} t+\psi\right)
$$

where $\psi$ is the phase error between the magnetron output and the injection signal, and $V_{\mathrm{RF}}$ is the circuit voltage associated with the RF power delivered by the magnetron. If now the injection signal is given a sudden phase shift $\phi$, i.e., $V_{\text {inj }} \cos \left(\omega_{i} t\right) \rightarrow$ $V_{\text {inj }} \cos \left(\omega_{i} t+\phi\right)$, then $\ddot{V}$ must instantaneously change to ensure that the circuit (1) is balanced. Changing $\ddot{V}$ immediately changes the period and hence the frequency, although $\omega_{o}$ and $\omega_{i}$ remain unchanged. David [19] derives the Adler equation [22]

$$
\frac{d \psi}{d t}=-\frac{V_{\mathrm{inj}}}{V_{\mathrm{RF}}} \frac{\omega_{o}}{2 Q_{L}} \sin \psi+\omega_{o}-\omega_{i}
$$

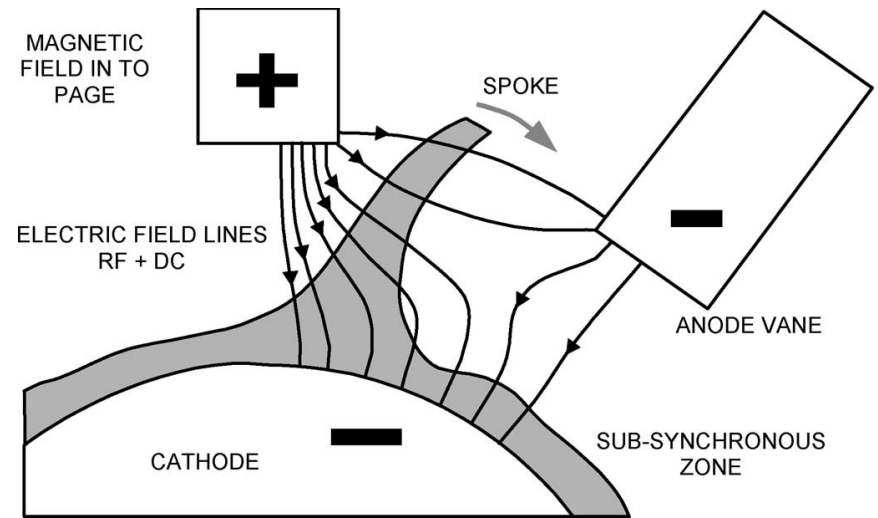

Fig. 9. Diagram showing the magnetron spoke position referenced to anode vane behind the spoke at the instant that vane takes its peak positive charge.

from (1) by assuming in (2) that $\psi$ and $V_{\mathrm{RF}}$ are slowly varying functions of time. The derivation requires one to neglect second derivatives, products of two first derivatives, and products of first derivatives with $\left(\omega_{o} / Q_{L}\right)\left(\left(Y_{e} / Y_{L}\right)-1\right)$. The Adler equation predicts that phase locking has a first-order response with a time constant given by $\left(Q_{L} / \omega_{o}\right) \sqrt{P_{\mathrm{RF}} / P_{\mathrm{inj}}}$, where $P_{\mathrm{RF}}$ is the RF power output that varies with $V_{\mathrm{RF}}^{2}$ and $P_{\text {inj }}$ is the injection power that varies as $V_{\mathrm{inj}}^{2}$. It also predicts that the response for advancing and retarding the phase is symmetric. For the magnetron used here, the loaded $Q_{L}$ was close to 100 . The injection power for the figures presented above was typically $1 \mathrm{~W}$, and the output power was typically $1000 \mathrm{~W}$; hence, the anticipated time constant for changing the phase is $400 \mathrm{~ns}$. Some of the traces in Figs. 5 and 6 show response times close to $200 \mathrm{~ns}$ and also that the response is second order and asymmetric. Instead of using the Adler equation to estimate the response, one can solve (1) numerically after making some assumption on the dependence of the electronic admittance on the power output. If one sets $\left(Y_{e} / Y_{L}\right)-1=1-\left(V^{2} / V_{\text {sat }}^{2}\right)$, where $V_{\text {sat }}$ is the steady-state RF output voltage, one obtains the Van der Pol oscillator [23]. Solving this case numerically, the response time for a $90^{\circ}$ shift is nearer to $600 \mathrm{~ns}$ than $400 \mathrm{~ns}$ as given by the Adler equation.

It is well known that the magnetron shifts its frequency by pushing effects as well as by pulling effects. We postulate that the discrepancy in the response occurs through the mechanism that also explains the magnetron's pushing characteristic [24]. The magnetron can increase or decrease its output frequency by changing the capacitive coupling between the spokes and the anode vanes. This happens as the spokes move to or away from the positively charged vanes referenced to a phase when the vane carries its maximum positive charge. A movement toward the vane increases the capacitance and decreases the output frequency, whereas a movement away from the vane decreases the capacitance and increases the output frequency (see Fig. 9).

As the spokes advance toward the midpoint between the vanes, the electrons in the spokes see a stronger circumferential retarding force, the RF power output increases, and the radial current flow through the spokes increases. The increase in the current is of course dependent on the ability of the power supply and cathode to deliver it. If the power supply or the cathode cannot respond, then either the subsynchronous zone shrinks or charge in the spokes is reduced, both outcomes resulting in 


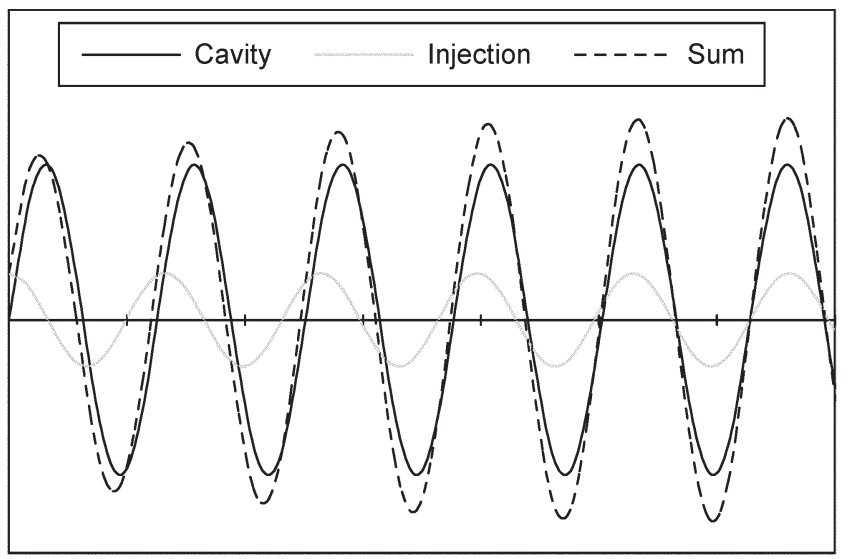

Fig. 10. Effect of an injection signal, horizontal axis is time.

the capacitance not increasing, as it should. This explanation is consistent with Brown's observations on the magnetron's response to steps in the anode voltage [9].

For a magnetron to generate power, the spokes must remain synchronized to the pi-mode. Synchronization occurs because as a spoke gets closer to the positively charged vane behind it at the phase of peak positive charge (apppc), it sees an increasing radial field that increases the circumferential drift velocity. (The drift velocity is the average velocity of the cycloidal motion of the electrons determined by $E \times B / B^{2}$.) There is a position of the spokes forward from the positively charged vane (apppc) where the circumferential drift velocity of electrons in the spokes matches the phase velocity of the pi-mode. Moving back from this point, the circumferential drift velocity increases, whereas moving forward of this point, the circumferential drift velocity reduces.

A full description of the locking mechanism for magnetrons requires consideration of how the spokes adjust the frequency and phase of oscillations in the magnetron cavities in response to the injection signal. Figs. 7 and 8 of the magnetron output phase against time show that the magnetron output frequency takes a large step change at the instant that the injection signal takes a new phase. The new magnetron output frequency is experimentally deduced from the rate of change of phase added to the double-balanced mixer reference frequency, which, in this case, is the magnetron output frequency when the phase is not being switched. Close inspection of the figures indicates that after the initial large jump in frequency, the frequency difference continues to increase very slightly, taking its maximum value well into the phase correction.

The contribution to the injection-locking mechanism from pushing effects can be explained with reference to Fig. 10.

Fig. 10 shows hypothetical potentials in the output cavity as a function of time. It shows the injection potential as a grey line, the total potential as a dashed line, and the total potential minus the injection potential (the cavity potential) as a solid black line. The injection signal is initially advanced by $90^{\circ}$ from the cavity oscillation. (It is drawn here with a much larger amplitude than is typically used for clarity of description.) The resultant field is very slightly advanced from the original oscillation. This means that the spokes have not got as far at the instant when the positive charge on the vane immediately behind the spoke is at its maximum. The capacitance is increased, and hence, the magnetron output frequency is decreased from the outset. (With reference to (1), $\omega_{o}$ changes, and hence, there is an immediate change in $\ddot{V}$.) Fig. 10 shows the cavity potential at a lower frequency than the injection potential. The new relative position for the spoke should also allow a step change in the current, although this change is not seen as a sharp step in the external circuit by our oscilloscope. Because the magnetron anode circuit is oscillating at a lower frequency than the injection signal, its phase retards to that of the injection signal.

With the spokes in an earlier position, electrons in the spoke see a smaller retarding field, and hence, the radial drift velocity is reduced. This will cause the charge density of the spoke to increase. This is possibly why the frequency difference continues to increase slightly before it decreases. The spoke is also in a large radial field and hence will have an increased circumferential drift velocity that starts to move it back to its equilibrium position. Of course, this position depends on the frequency of the magnetron pi-mode at any instant and is not back to its original value until the phase shift is complete. As yet, we do not have a good model for the dynamic response of the spokes. At high heater powers, the data suggest that the spokes may oscillate about their equilibrium position, causing the frequency and phase to oscillate as the capacitance changes. It should be noted that the magnetron models of Hull [25], Vaughan [26], and Riyopoulos [27] all fix the phase of spoke with respect to the electromagnetic wave such that the dc power input to the magnetron equals the RF output plus losses. In those papers, no attempt is made to find the phase of the spoke from the steady-state solution of a dynamic model.

How the current changes at the instant the relative position of the spokes changes has a dependence on the external highvoltage dc circuit and stored charge in the subsynchronous zone. Figs. 7 and 8 suggest that it is easier for the magnetron spokes to draw less current than it is for them to draw more current when a change of the injection signal phase is made. This effect may also occur just because the circumferential electric field is not changing linearly with angle near to the position of the spoke. Asymmetry of the switching response is likely to be a combination of this factor and the availability of charge.

One might suppose that the switching time can be estimated from the change in the anode current. The switching time should be given by the phase shift divided by the shift of the angular frequency, i.e.,

$$
t_{\mathrm{switch}}=\frac{\Delta \phi}{\Delta \omega} .
$$

The frequency shift can be determined from the change in the anode current using constant temperature pushing curves. (These curves are steeper than those measured when the anode temperature is allowed to increase as the anode current increases.) From [4], the frequency shift for a change in anode current from 300 to $200 \mathrm{~mA}$ is $2 \mathrm{MHz}$; hence, for the case where the anode current changes by this amount and the phase shift is $90^{\circ}$ (e.g., the case of Figs. 7 and 8), then

$$
t_{\mathrm{switch}}=\frac{\pi}{2} \times \frac{1}{2 \pi \times 2 \times 10^{6}}=125 \mathrm{~ns} .
$$




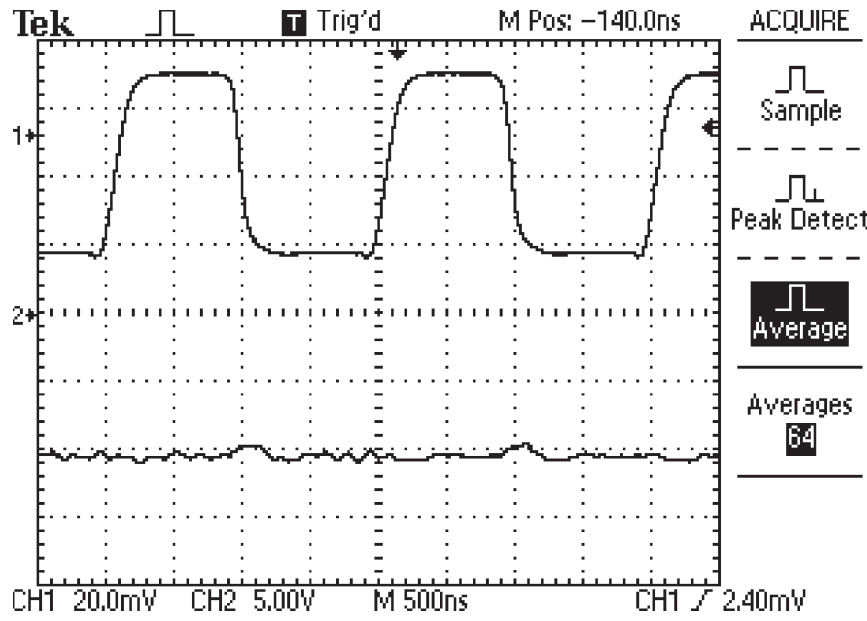

Fig. 11. Anode current (lower trace) referenced to the magnetron phase (upper trace) for $90^{\circ}$ phase shifts, a heater power of $15 \mathrm{~W}$, an injection power of $1 \mathrm{~W}$, and a matched load. Average current $=220 \mathrm{~mA}$, frequency $=2.4506 \mathrm{GHz}$.

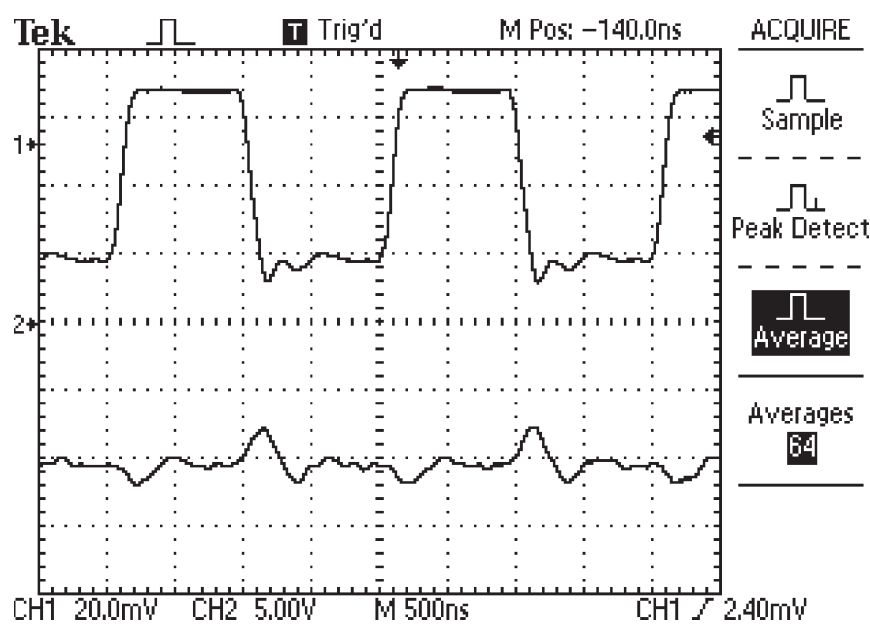

Fig. 12. Anode current (lower trace) referenced to the magnetron phase (upper trace) for $90^{\circ}$ phase shifts, a heater power of $36 \mathrm{~W}$, an injection power of $1 \mathrm{~W}$, and a matched load. Average current $=219 \mathrm{~mA}$, frequency $=2.4506 \mathrm{GHz}$.

The actual switching time for retarding the phase for the case of Figs. 7 and 8 was about 200 ns and a little less than 200 ns, respectively.

Figs. 11-13 show the switching response and the associated current variation for differing operating parameters. Fig. 11 shows the response for a low heater power. Here, the switching time for retarding the phase is still about $200 \mathrm{~ns}$; however, the reduction in anode current is only $30 \mathrm{~mA}$. At first sight, estimation of the switching time as given above does not seem to work. The discrepancy occurs because the constant temperature pushing curves in [4] were determined for a high heater power of about $43 \mathrm{~W}$. In the next section, it will be seen that the pushing curves for low heater powers at low anode currents are much steeper, and it turns out that the estimation is still reasonable.

Anode current variations also become quite small for high anode currents at intermediate heater powers as illustrated in Figs. 12 and 13. For the case of Fig. 13, the current changes by about $30 \mathrm{~mA}$; hence, one estimates the switching time to be about $400 \mathrm{~ns}$ in approximate agreement with the data.



Fig. 13. As Fig. 12 except for average current $=363 \mathrm{~mA}$, frequency $=$ $2.4518 \mathrm{GHz}$

\section{Pushing Curves as a Function of Heater Power}

An interesting feature observed when making the measurements for Fig. 6 (see caption) is that as the heater power is increased from 8 to $43 \mathrm{~W}$, the current initially decreases before increasing again. For the magnetron under investigation, it became apparent that its properties changed abruptly at a heater current between 18 and $21 \mathrm{~W}$. This feature was investigated by determining pushing curves as a function of heater power. For a magnetron that is not injection locked, this is almost impossible to do because a free-running magnetron will have a range of heater powers and anode currents where the magnetron is so noisy that it is impossible to make meaningful frequency measurements. Indeed, for the magnetron under investigation, it was not possible to control its frequency as a current-controlled oscillator (and without injection) for heater powers between 15 and $30 \mathrm{~W}$. With injection and current control, it was possible to phase lock the magnetron for any heater power, and hence, pushing curves could be obtained. A point to note with respect to the experimental detail and related to our PLL control is that the injection level can be set too high for pushing curves to be recorded. Indeed, it was necessary to reduce the injection power for lower anode currents. The reason for this is that, at high injection levels, the phase error between the reference and the magnetron is so small that the PLL stops adjusting the current. This means that the current stays constant as the frequency is adjusted until the lock jumps (i.e., phase lock by the injection signal is lost for a few milliseconds or less while the PLL control loop readjusts the current).

Fig. 14 shows the pushing curves obtained for our magnetron. They were obtained by simultaneously incrementing the injection frequency and the PLL control frequency and measuring the anode current at constant heater power. The anode temperature was also measured. The experiments were performed in such a way that the magnetron anode was heated at roughly the same rate for each heater power. This was achieved by incrementing the frequency at the same rate for each curve (filament power constant) and then letting the anode cool back to the same temperature before the data set for next filament power was collected. Frequency data were then corrected for small 


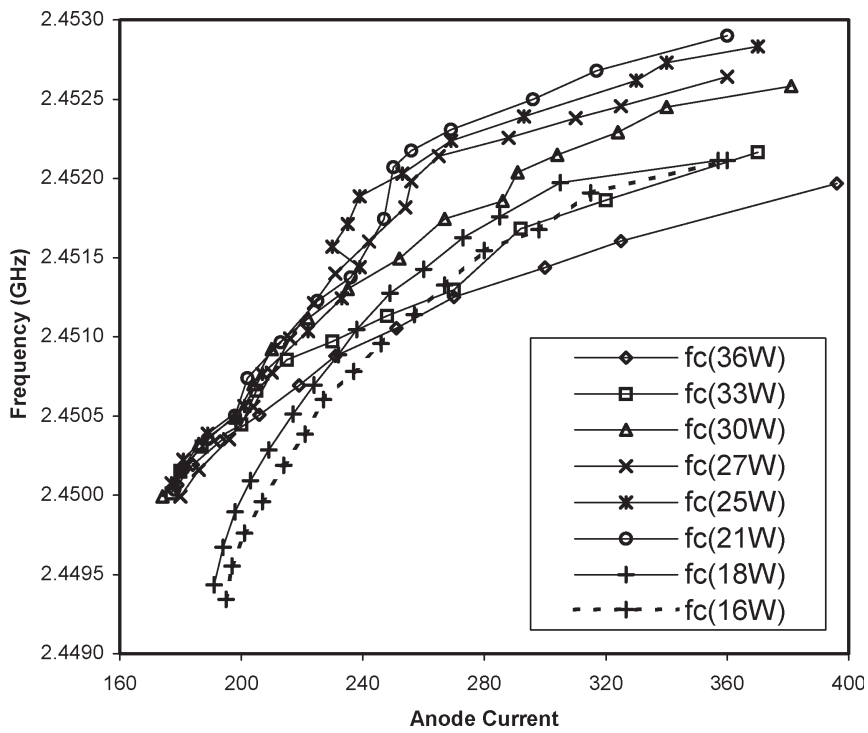

Fig. 14. Pushing curves as a function of heater power measured with an injection-locked magnetron and for a steadily increasing anode temperature with anode current.

temperature deviations from the mean temperature at each current. The frequency correction applied was $2 \mathrm{MHz}$ for $100{ }^{\circ} \mathrm{C}$ as derived from constant temperature pushing curves [4].

The pushing curves of Fig. 14 show that at lower heater powers, there are two distinct modes of operation. The crossover is at $20 \mathrm{~W}$ of heater power. Below $20 \mathrm{~W}$, it is likely that the subsynchronous zone is starved of electrons. The extent to which it forms depends crucially on secondary emission [9]. Above $20 \mathrm{~W}$ but at low anode currents, the subsynchronous zone grows until it is space charge limited. Noting that a high frequency implies a lower effective capacitance for the anode cavities, then one infers from Fig. 14 that increasing the heater power for powers above $20 \mathrm{~W}$ and for anode currents above $240 \mathrm{~mA}$, one has an increased capacitance. Intuitively, one supposes that as the heater power is increased in this regime, then either the radius of the subsynchronous zone grows or the charge density in the spokes grows (or both), both of which would increase the capacitance.

For low anode currents, the subsynchronous zone or the charge densities in the spokes grow to a limiting value.

It is reasonably clear that a magnetron being operated close to the transition heater power (in this case $20 \mathrm{~W}$ and whose frequency is not injection locked) would become very noisy or would exhibit twining [28]. The transition point would of course vary with depletion of thorium in a thoriated cathode. This type of noisy behavior of cooker magnetrons occurring at specific heater powers (filament voltages) is known and has been reported [11].

\section{FSK SWITCHING}

As well as PSK amplification by the magnetron, FSK amplification by the magnetron is also possible. Fig. 15 shows the output from the double-balanced mixer for an FKS input. The PLL control frequency for the magnetron was $2.452 \mathrm{GHz}$, the injection frequency was switched from 2.452 to $2.453 \mathrm{GHz}$

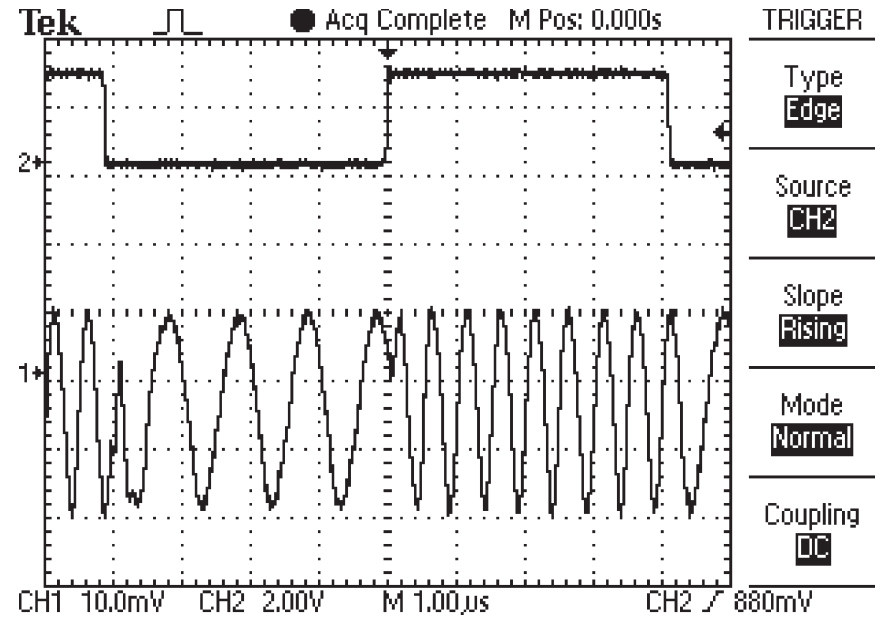

Fig. 15. Lower trace is the output from the double-balance mixer when the magnetron injection signal is switched from 2.452 to $2.453 \mathrm{GHz}$ at a rate of $250 \mathrm{~kb} / \mathrm{s}$ (upper trace) and referenced to $2.451 \mathrm{GHz}$.

at a rate of $250 \mathrm{~kb} / \mathrm{s}$, and the reference frequency for the doublebalanced mixer was $2.451 \mathrm{GHz}$.

The transition between the two frequencies depends on the level of $100-\mathrm{Hz}$ ripple at any instant and can take up to $1 \mu \mathrm{s}$. The figure was sampled at a time when the transition was very short.

\section{CONCLUSION}

The response of an injection-locked $\mathrm{CW}$ magnetron to changes in phase and frequency has been studied as a function of heater power. The response characteristic and time constant differ from what one would expect from Adler's theory that relies solely on a pulling effect; as well as being faster, it is differentially second order and asymmetric. These observations can be explained if it is assumed that the locking mechanism also depends on the same physical process, which leads to frequency pushing.

\section{ACKNOWLEDGMENT}

The authors would like to thank A. Verden of Lancaster University for practical assistance and Agilent for the loan of a DSO $80808 \mathrm{GHz}, 40 \mathrm{GS} / \mathrm{s}$ oscilloscope to independently check gain performance.

\section{REFERENCES}

[1] J. Kline, "The magnetron as a negative-resistance amplifier," IRE Trans. Electron Devices, vol. ED-8, no. 6, pp. 437-442, Nov. 1961.

[2] H. L. Thal and R. G. Lock, "Locking of magnetrons by an injected r.f. signal," IEEE Trans. Microw. Theory Tech., vol. MIT-13, no. 6, pp. 836-846, Nov. 1965

[3] M. H. Seavey, Jr., "Some properties of an injection locked pulsed magnetron in a coherent-echo-detection system," Electron. Lett., vol. 3, no. 8, pp. 375-377, Aug. 1967.

[4] I. Tahir, A. C. Dexter, and R. G. Carter, "Noise performance of frequency and phase locked $\mathrm{CW}$ magnetrons operated as current controlled oscillators," IEEE Trans. Electron Devices, vol. 52, no. 9, pp. 2096-2103, Sep. 2005.

[5] N. Shinohara, H. Matsumoto, and K. Hashimoto, "Solar power station/ satellite (SPS) with phase controlled magnetrons," IECE Trans. Electron., vol. E86-C, no. 8, pp. 1550-1555, Aug. 2003. 
[6] R. D. Weglein and H. A. Leach, "The noise behavior of an injection locked magnetron reflection amplifier," in Proc. IEEE MTT-S Int. Microwave Symp., 1987, pp. 261-264.

[7] W. C. Brown, "A reporting of personal observations of noise in magnetrons and CFAs," Raytheon Company, Waltham, MA, Raytheon Internal Rep. PRP-5504, Mar. 1988.

[8] — , "The history of the reentrant beam crossed field amplifier with emphasis on noise comparison with the magnetron," in Proc. 1st Int. Workshop Cross-Field Devices, Aug. 1995, pp. 9-22.

[9] _- "The high signal to noise ratio of the microwave oven magnetron and evidence of a negative feedback loop to control it," in Proc 1st Int. Workshop Cross-Field Devices, Aug. 1995, pp. 178-187.

[10] W. E. Garrigus, "Phase noise performance of magnetrons," Microw. J., vol. 20, pp. 59-73, Jul. 1977.

[11] J. M. Osepchuk, "The cooker magnetron as a standard in crossed-field device research," in Proc. Ist Int. Workshop Crossed-Field Devices, Ann Arbor, MI, Aug. 15-16, 1995, pp. 157-159.

[12] T. Mitani, N. Shinohara, H. Matsumoto, M. Aiga, N. Kuwahara, and T. Handa, "Time domain analysis of noises generated from microwave oven magnetron," Electron. Commun. Jpn., vol. 88, pt. 2, no. 10, pp. 28-36, 2005.

[13] V. B. Neculaes, R. M. Gilgenbach, Y. Y. Lau, M. C. Jones, and W. M. White, "Low-noise microwave oven magnetrons with fast startoscillation by azimuthally varying axial magnetic fields," IEEE Trans. Plasma Sci., vol. 32, no. 3, pp. 1152-1159, Jun. 2004.

[14] V. B. Neculaes, M. C. Jones, R. M. Gilgenbach, Y. Y. Lau, J. W. Luginsland, B. W. Hoff, W. M. White, N. M. Jordan, P. Pengvanich, Y. Hidaka, and H. L. Bosman, "Magnetic perturbation effects on noise and startup in DC-operating oven magnetrons," IEEE Trans. Electron Devices, vol. 52, no. 5, pp. 864-871, May 2005.

[15] _ _Magnetic priming effects on noise, startup, and mode competition in magnetrons," IEEE Trans. Plasma Sci., vol. 33, no. 1, pp. 94-102, Feb. 2005.

[16] T. Overett, D. B. Remsen, E. Bowles, G. E. Thomas, and R. E. Smith, "Phase locked magnetrons as accelerator sources," in Proc. PAC, 1987, pp. 1464-1465.

[17] S. C. Chen, G. Bekefi, and R. J. Temkin, "Injection locking of a long-pulse relativistic magnetron," in Proc. PAC, 1991, pp. 751-753.

[18] T. A. Treado, R. A. Bolton, T. A. Hansen, P. D. Brown, and J. D. Barry, "High-power, high efficiency, injection-locked secondary-emission magnetron," IEEE Trans. Plasma Sci., vol. 20, no. 3, pp. 351-359, Jun. 1992.

[19] E. E. David, "RF phase control in pulsed magnetrons," Proc. IRE, vol. 40, pp. 669-685, Jun. 1952.

[20] W. Woo et al., "Phase locking of high power microwave oscillators," J. Appl. Phys., vol. 65, no. 2, pp. 861-866, Jan. 1989.

[21] J. C. Slater, "The phasing of magnetrons," Res. Lab. Electron., MIT, Cambridge, MA, Tech. Rep. 35, 1947.

[22] R. Adler, "A study of locking phenomena in oscillators," Proc. IRE, vol. 34, pp. 283-284, Jun. 1946.

[23] B. Van der Pol, "The nonlinear theory of electric oscillations," Proc. IRE, vol. 22, no. 9, pp. 1051-1086, 1934.

[24] H. W. Welch, S. Ruthberg, H. W. Batten, and W. Peterson, "Analysis of dynamic characteristics of the magnetron space charge preliminary results," Electron Tube Lab., Dept. Elect. Eng., Univ. Michigan, Ann Arbor, Tech. Rep. 5, 1951

[25] J. F. Hull, "Crossed field electron interaction in space charge limited beams," Ph.D. dissertation, Dept. Elect. Eng., Polytechnic Inst., Brooklyn, NY, Jun. 1958.

[26] J. R. M. Vaughan, "A model for calculation of magnetron performance," IEEE Trans. Electron Devices, vol. 20, no. 9, pp. 818-826, Sep. 1973.
[27] S. Riyopoulos, "New improved formulas for magnetron characteristic curves," IEEE Trans. Plasma Sci., vol. 26, no. 3, pp. 755-766, Jun. 1998.

[28] G. R. Carter, E. M. Ball, and M. Brady, "Magnetron frequency twinning," IEEE Trans. Plasma Sci., vol. 28, no. 3, pp. 905-909, Jun. 2000.

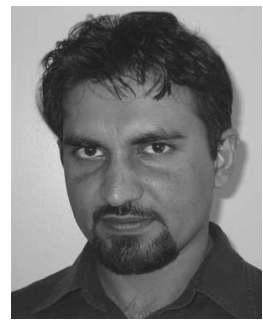

Imran Tahir was born in Burewala, Pakistan, in 1977. He received the B.E. (Hons.) degree from the University of Engineering and Technology, Lahore, Pakistan. He is currently working toward the Ph.D. degree in microwave electronics at Lancaster University, Lancaster, U.K.

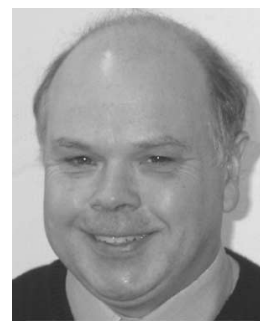

Amos Dexter was born in Carshalton, U.K., in 1956. $\mathrm{He}$ received the B.Sc. (Hons.) degree in mathematics and physics from Bristol University, Bristol, U.K., in 1978 and the Ph.D. degree in quantum field theory from Liverpool University, Liverpool, U.K., in 1982

In 1981, he joined the Electricity Council Research Centre, Capenhurst, U.K., where he specialized in electromagnetic heating and plasma processing. In 2002, he took up a lectureship with the Engineering Department at Lancaster University, Lancaster, U.K. He is the Director of Studies for a Joint M.Sc. with Strathclyde University on High Power Radio Frequency Science and Engineering. His current research interests include microwave tube development, radio frequency (RF) cavities, RF systems, and multipactor.

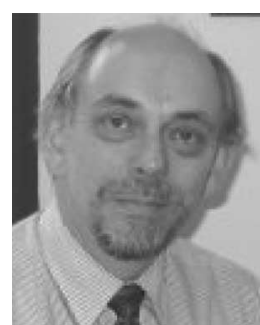

Richard Carter (M'97-SM'01) received the B.A (Hons.) degree in physics from the University of Cambridge, Cambridge, U.K., in 1965 and the Ph.D. degree in electronic engineering from the University of Wales, Wales, U.K., in 1968 for work on the propagation of waves on neutralized ion beams.

From 1968 to 1972, he worked on high-power travelling-wave tubes as a Development Engineer at English Electric Valve Company Ltd. He joined the Engineering Department of the University of Lancaster, Lancaster, U.K., as a Lecturer in 1972 and was promoted to Senior Lecturer in 1986 and Professor of electronic engineering in 1996. He is the author of a number of papers, two textbooks on electromagnetics, and a set of video lectures on microwave tubes. He has played key roles in the establishment of the Faraday Partnership in High Power RadioFrequency Engineering and of the Cockcroft Institute (International Centre for Accelerator Science and Technology). His research interests include electromagnetics and microwave engineering with particular reference to the theory, design, and computer modeling of microwave tubes and particle accelerators.

Prof. Carter is a Fellow of the Institution of Electrical Engineers. 